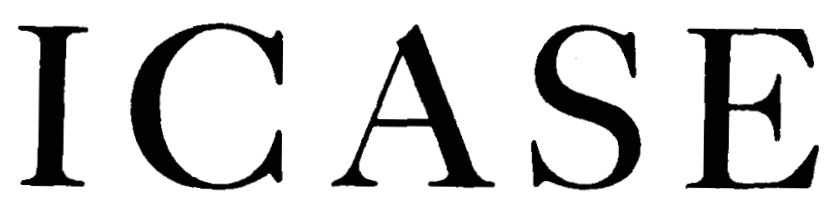

ON THE PREDICTION OF EQUILIBRIUM STATES IN HOMOGENEOUS TURBULENCE

Charles G. Speziale

Nessan Mac Giolla Mhuiris

(HASA-CR-181659) ON THE PFBDTCETON OH

EQUILIURIUM STATES IN HONOGENEOUS TUGBUL BNL

$\operatorname{No8}-23174$

Final feport

(NASA)

$+7 \mathrm{p}$

CSCL

Inclas

Contract No. NAS $1-18107$

$63 / 34 \quad 0142688$

Apr11 1988

INSTITUTE FOR COMPUTER APPLICATIONS IN SCIENCE AND ENGINEERING

NASA Langley Research Center, Hampton, Virginia 23665

Operated by the Universities Space Research Association

\title{
NMSA
}

National Aeronautics and

Space Administration 
NASA Contractor Report 4144

\section{Secondary Instability \\ of Compressible Boundary \\ Layer to Subharmonic \\ Three-Dimensional Disturbances}

Nabil M. El-Hady

Old Dominion University

Norfolk, Virginia

Prepared for

Langley Research Center

under Grant NAG1-729

\section{NMSA}

National Aeronautics

and Space Administration

Scientific and Technical

Information Division 
SECONDARY INSTABILITY OF COMPRESSIBLE BOUNDARY LAYER

TO SUBHARMONIC THREE-DIMENSIONAL DISTURBANCES

Nabil M. E1-Hady

Department of Mechanical Engineering and Mechanics

01d Dominion University, Norfolk, Virginia 23508

ABSTRACT

Three-Dimensional linear secondary instability theory is extended for compressible boundary layers on a flat plate in the presence of finite amplitude Tollmien-Schlichting $(\mathrm{T}-\mathrm{S})$ wave. The focus is on principal parametric resonance responsible for strong growth of subharmonics in low disturbance environment.

\section{INTRODUCTION}

Whatever theoretically or experimentally, the compressible stability theory lack a firm connection with boundary-layer transition."There is little doubt that transition is preceded by linear instability in many instances, but the way these Individual unstable waves act, alone or in combination, to trigger the transition process is not known" [1]. The nonlinear theories and the secondary instability that are much a prominent feature of incompressible stability theory do not exist for compressible boundary layer. 
Recently, Erlebacher and Hussaini $[2,3]$ by using direct simulation of parallel boundary layer, generated numerically a high Mach number vortical structure (peak-vally) similar to that observed and computed for incompressible flows.

Stimulated by this work, we formulated the secondary three-dimensional instability problem for compressible boundary layers. In this paper, we investigate theoretically the effect of finite amplitude two-dimensional (2D) wave on the growth of three-dimensional (3D) perturbations in compressible boundary layers. Hence, this paper covers only a range of Mach numbers up to the transonic, where the critical primary disturbance is 2D. For supersonic boundary layers, the critical primary disturbance is $3 \mathrm{D}$, or a $2 \mathrm{D}$ second mode. This range of Mach numbers is currently under investigation. An analysis similar to that of Herbert [4], Herbert et al [5] and Nayfeh [6] is followed here but spatial stability is considered for both primary and secondary instabilities.

The primary instability leads to the growth of $\mathrm{T}-\mathrm{S}$ waves and a streamwise almost periodic modulation of the flow. We study the linear stability of this flow with respect to spanwise periodic 3D disturbence. Floquet theory gives as a solution to the stability equations, all various types of resonance. We consider the case of principal parametric resonance responsible for strong growth of subharmonics in low disturbance environment.

II. PRIMARY INSTABILITY AND THE BASIC FLOW 
We cosider the primary instability of the 2D compressible boundary layer on a flat plate, with respect to 2D quasi-parallel spatially growing $T-S$ disturbances. The critical disturbance is 2D up to the transonic range of Mach numbers [7]. The flow field is governed by the 2D compressible Navier-Stokes equations, the energy equation and the state equation. Dimensionless quantities are introduced by using the reference velocity $U_{r}$ and the referece length $L=\sqrt{\nu x / U_{r}}$, so that Reynolds number is given by $R=\sqrt{U_{r} x / \nu}$, where $x$ measures the distance from the leading edge of the plate, and $\boldsymbol{\nu}$ is the fluid kinematic viscosity. The thermodynamic and transport properties of air (treated as perfect gas) are made dimensionless using their corresponding freestream values.

At sufficiently large distance from the leading edge, primary instability of the compressible laminar flow occurs with respect to T-S disturbances. These disturbances take the traveling wave form,

$$
\begin{aligned}
\left(u_{p}, v_{p}, p_{p}, t_{p}, \mu_{p}, p_{p}\right)=A_{0}[ & u(y), v(y), p(y), t(y), \mu(y), p(y)] \\
& \exp \left[i\left(\int \alpha d x-\omega t\right)\right]+c \cdot c .
\end{aligned}
$$

Where the $y$-axis is defined normal to the flat plate, $u$ and $v$ are the disturbance velocities, $p$ is the disturbance pressure, $t$ is the disturbance temperature, $\mu$ is the disturbance viscosity, and $\rho$ is the disturbance density.

For the spatial stability analysis $\alpha$ is a complex wavenumber given by $\alpha=\alpha_{r}+1 \alpha_{i}$ and $\omega$ is a real disturbance frequency, and c.c. denotes complex conjugate terms. The eigensolutions $u, v, p$ and $t$ are governed by $a$ six-order system of equations that is given in reference (9). The density disturbance is 
related to the temperature and pressure disturbances through the state equation,

$$
\gamma_{M_{\infty} p}^{2}=\rho T+\bar{p} t
$$

while the viscosity disturbance is related to the temperature disturbance by

$$
\mu=(d \bar{\mu} / d T) t=\hat{\mu} t
$$

where $\bar{\rho}$ and $\bar{\mu}$ are the mean-flow density and viscosity respectively, $Y$ is the ratio of specific heats and $M_{\infty}$ is the freestream Mach number. The six-order system is numerically integrated as initial value problem using a combination of shooting [8] and Newton-Raphson iteration technique that employs a Gram-Schmidt orthonormalization procedure. The solution of this eigenvalue problem is calculated by the author elsewhere $[7,9]$.

The linear stability theory of primary instability provides $\alpha$ for a given $\omega$ and $R$. Then the integration of the growth rate $-\alpha_{i}$ gives the amplification factor,

$$
\ln \left(A / A_{0}\right)=-2 \int_{R_{o p}}^{R} \alpha_{i} d R
$$

where $A_{0}$ is an arbitrary initial amplitude of the primary instability at $R_{\text {op }}$ ( $R$ where the onset of the primary wave). The eigensolutions may be normalized such that A measures directly the maximum r.m.s. value of the streamwise disturbance, that is

$$
\max _{0 \leqslant y<\infty}|u(y)|^{2}=1.2
$$

Since the primary instability of boundary-layer flows is induced by viscosity, the growth rates and amplification factors here are typically very small. 
Now the basic state under consideration is composed of the sum of the 2D compressible mean flow and a finite amplitude $A$ of the primary $T-S$ wave, that is

$$
\left(u_{b}, v_{b}, p_{b}, t_{b}\right)=(U, 0, P, T)+A[u(y), v(y), p(y), t(y)] \exp (i \theta)
$$

Where

$$
\begin{aligned}
& A=A_{0} \exp \left(-\int \alpha_{i} d x\right), \quad \text { assumed constant, and } \\
& \theta=\int \alpha_{r} d x-\omega t
\end{aligned}
$$

\section{SECONDARY SUBHARMONIC INSTABILITY}

We consider the 3D quasi-parallel spatial subharmonic instability of the basic state given by (6). The finite amplitude primary wave acts as a parametric exitation on the secondary instability. Following the analysis of Herbert [4,5], Nayfeh [6], and El-Hady [10] we apply Floquet theory and express the secondary wave using the normal mode concept,

$$
\left(u_{s}, v_{s}, p_{s}, t_{s}\right)=\exp \left(\int \hat{\gamma} d x\right)[\hat{u}(y), \hat{v}(y), \hat{p}(y), \hat{t}(y)] \exp \left(\frac{1}{2} i \theta\right) \cos \beta z
$$

$$
w_{s}=\exp \left(\int \hat{\gamma} d x\right) \hat{w}(y) \exp \left(\frac{1}{2} i \theta\right) \sin \beta z+c \cdot c .
$$

where $\beta$ is a spanwise real wavenumber, and $\hat{\gamma}=Y_{r}+i \gamma_{i}$ is a characteristic exponent. The spatial growth rate of the secondary wave is given by $\gamma_{r}$, while $Y_{i}$ can be interpreted as a shift in the streamwise wavenumber. In our calculations, we consider only the case of $\gamma_{i}=0$, that is the secondary wave is perfectly synchronized with the basic state. 
The secondary wave (7) is superposed on the basic state (6) and the result is substituted into the dimensionless Navier-Stokes equations. The meam flow plus the 2D T-S quantities are substracted, and the resulting equations are linearlzed in the secondary disturbances. Then one obtains an eigenvalue problem that can be written as

$$
\begin{aligned}
& T^{-1}\left(g Z_{1}-T^{-1} D T Z_{3}+D Z+\beta Z_{4}\right)+G\left(\gamma M_{\infty}^{2} z_{6}-T^{-1} Z_{7}\right) \\
& \quad+A\left(a_{1}\right)=0
\end{aligned}
$$

$$
\begin{aligned}
& \left\{G-\bar{\mu} R^{-1}\left[(m+2) g^{2}-\beta^{2}\right]\right\} z_{1}-R^{-1} D\left(\bar{\mu} z_{2}\right) \\
& \quad+\left(T^{-1} D U-D \bar{\mu} R^{-1} g\right) z_{3}-(m+1) \bar{\mu} R^{-1} g\left(D z_{3}+\beta z_{4}\right) \\
& +g z_{6}-R^{-1} D(\hat{\mu} D U) z_{7}-\hat{\mu} R^{-1} D U z_{8}+A\left(a_{2}\right)+A^{2}\left(b_{2}\right)=0
\end{aligned}
$$

\section{$(10)$}

$$
\begin{aligned}
& {\left[G-\bar{\mu} R^{-1}\left(g^{2}+\beta^{2}\right)\right] z_{3}-(m+1) \bar{\mu} R^{-1}\left(g z_{2}+\beta z_{5}\right)} \\
& -(m+2) R^{-1} D\left(\bar{\mu} D z_{3}\right)-m R^{-1} D \bar{\mu}\left(g z_{1}+\beta z_{4}\right)+D Z_{6} \\
& -\hat{\mu} R^{-1} D U g z_{7}+A\left(a_{3}\right)+A^{2}\left(b_{3}\right)=0
\end{aligned}
$$

(11) $\quad(m+1) \bar{\mu} R^{-1} \beta\left(g z_{1}+D z_{3}\right)+D \bar{\mu} R^{-1} \beta z_{3}$

$$
\begin{aligned}
& {\left[G-\bar{\mu} R^{-1} g^{2}+(m+2) \bar{\mu} R^{-1} \beta^{2}\right] z_{4}-R^{-1} D\left(\bar{\mu} z_{5}\right)} \\
& -\beta z_{6}+A\left(a_{4}\right)+A^{2}\left(b_{4}\right)=0
\end{aligned}
$$


(12)

$$
\begin{aligned}
& -2 \hat{M} \hat{\mu} R^{-1} \text { DU }\left(z_{2}+g z_{3}\right)+T^{-1} \text { DT } z_{3}-\hat{M} \text { T G } z_{6} \\
& +\left\{G-R^{-1} \operatorname{Pr}^{-1}\left[\bar{\mu}\left(g^{2}-\beta^{2}\right)+D(\bar{\mu} D T)\right]-\hat{M} R^{-1} \text { (DU) } \hat{\mu}\right\} z_{7} \\
& -R^{-1} \operatorname{Pr}^{-1}\left(2 Z_{8} D \bar{\mu}-\bar{\mu} D z_{8}\right)+A\left(a_{5}\right)+A^{2}\left(b_{5}\right)=0
\end{aligned}
$$

where

$$
\begin{aligned}
& z_{1}=\hat{u}, z_{2}=\hat{D u}, z_{3}=\hat{v}, z_{4}=\hat{w}, \\
& z_{5}=\hat{D w}, z_{6}=\hat{p}, z_{7}=\hat{t}, z_{8}=\hat{D t} \\
& g=\left(Y_{r}+\frac{1}{2} i \alpha_{r}\right) \\
& G=T^{-1}\left(g U-\frac{1}{2} i \omega\right) \\
& \hat{M}=(Y-1) M_{\infty}^{2}
\end{aligned}
$$

also $\operatorname{Pr}$ is Prandt1 number, $D=d / d y$, and $m=2(e-1) / 3$ is the ratio of the coefficients of viscosities, where $e=0$ corresponds to the Stokes hypothesis.

The boundary conditions are

$$
z_{1}=z_{3}=z_{4}=z_{7}=0 \quad \text { at } y=0
$$

$$
z_{1}, z_{3}, z_{4}, z_{7} \rightarrow 0 \quad \text { as } y \rightarrow \infty
$$


Equations (8)-(12) govern the secondary 3D instability of compressible 2D flows. They represent the mass, $x$-momentum, y-momentum, $z$-momentum, and energy equations respectively. The coefficients of $A$ and $A^{2}\left(a_{i}, b_{i}, 1=1-5\right)$ are given in Appendix A. The coefficients of $A$ in the mass equation and those of $A^{2}$ in a11 other equations are new in this system of equations in contrast to the secondary instability equations for incompressible flows. These coefficients are present mainly due to the density disturbance of both primary and secondary instabilities. In the incompressible limit eqations (8)-(12) reduce exactly to that given by E1-Hady [10]. When $A=0$, the system of equations (8)-(13) govern a primary subhamonic 3D wave.

The system of equations (8)-(12) can be written as eight complex equations in the form,

$$
\begin{aligned}
& D Z_{1}-z_{2}=0 \\
& \mathrm{DZ}_{2}+\mathrm{c}_{0} \overline{\mathrm{DZ}}_{2}+\mathrm{c}_{1}=0 \\
& \mathrm{DZ}_{3}+\mathrm{c}_{2} \overline{\mathrm{D}}_{3}+\mathrm{c}_{3}=0 \\
& D z_{4}-z_{5}=0 \\
& \mathrm{DZ}_{5}+\mathrm{c}_{4} \overline{D Z}_{5}+\mathrm{c}_{5}=0 \\
& \mathrm{DZ}_{6}+\mathrm{c}_{6} \overline{\mathrm{DZ}}_{6}+\mathrm{c}_{7}=0 \\
& \mathrm{DZ}_{7}-\mathrm{z}_{8}=0 \\
& \mathrm{DZ}_{8}+\mathrm{c}_{8} \mathrm{D \overline {Z } _ { 8 }}+\mathrm{c}_{9}=0
\end{aligned}
$$

Where the $c^{-} s$ are quadratures in the primary and secondary disturbance 
quantities, and the overbar indicates a complex conjugate. Equations (14) are numerically integrated as initial value problem from $y=y_{e}$ (edge of the boundary layer) to the wall. The eigenvalue search used a Newton-Raphson iteration technique to satisfy the last boundary condition at the wall. A well tested code SUPORT [8] is used which is coupled with an orthonormalization test based on the modefied Gram-Schmidt procedure to overcome the stiffness of the integrated system of equations.

The linear stability theory of the secondary instability provides $\hat{\gamma}$ for a given $\beta$ and $R$. Then the integration of the growth rate $\gamma_{r}$ gives the amplification factor,

$$
\ln \left(s / s_{0}\right)=2 \int_{R_{0 S}}^{R} \gamma_{r} d R
$$

where $S_{0}$ is an arbitrary initial amplitude of the secondary instability at $R_{0 S}$ ( $R$ where the onset of the secondary wave). The secondary subharmonic instability is believed to originate from a strong mechanism of combined tilting and stretching of the vortices such as the case of incompressible flows [10], thus the growth rates and amplification factors are expected to be large as they occur on a convective length scale.

IV. RESULTS AND DISCUSSION

For the incompressible limit $\left(M_{\infty}=0\right)$, our compressible secondary Instability code gives results that are in full agreement with those obtained by Herbert et al.[5] and by E1-Hady [10]. All results reported here are for the 
nondimensional frequency $F=\omega / R=60 \mathrm{E}-6$, that remains fixed as a wave of fixed physical frequency travels downstream.

At $M_{\infty}=0$, a primary 2D instability grows between $R_{o p}=554$ and $R_{1 p}=1052$ (first and second neutral points) reaching a maximum amplification factor of $\mathrm{A} / \mathrm{AO}=41.68$. As Mach number increases, the growth rates of the primary 2D waves decrease as shown in Fig.(1). Also the first and second neutral points as well as the streamwise location where the maximum growth rate occurs shift to the left, they occur earlier upstream as Mach number increases. Fig.(2) shows a reduction in the amplification factor of the primary $2 \mathrm{D}$ instability as Mach number increases.

Almost in the same region where the primary $2 \mathrm{D}$ waves are growing, a broad band of spanwise wavenumbers of primary 3D subharmonic waves are subject to amplification. Fig.(3) shows the growth rate curves of these primary 3D subharmonics $(F=30 \mathrm{E}-6)$ for different Mach numbers. These curves possess the same features of the primary 2D waves of having lower growth rates that shift to lower $R$ as Mach number increases. Fig.(4) shows the amplification factors of these primary subharmonics. Both Figs.(3) and (4) suggest that the growth rates and amplification factors of the primary subharmonics are typically so small to bear any resemblence to experimentally observed transition.

However, the growth rates shown in Fig.(5) as function of the spanwise wavenumber $B=10 \mathrm{E}+3 \beta / \mathrm{R}$ for secondary $3 \mathrm{D}$ subharmonics, are much larger than those for primary 2D waves or primary 3D subharmonics. This strong growth is due to the parametrical exitation by the finite amplitude primary 2D wave. 
Fig. (5) is calculated at $R=850$ and a primary $2 D$ amplitude $A=.01$, for different Mach numbers. For high spanwise wavenumbers $(B \geqslant .225)$, compressibility appears to have a stabilizing effect on the secondary subharmonic instability. In other words, the secondary subharmonic instability is largest at $M_{\infty}=0$, and decreases as Mach number increases. But for low spanwise wavenumbers $(B<.225)$ the effect of compressibility depends on the value of the spanwise wavenumber.

At fixed $F$ and $R$, results for different Mach numbers show a destabilizing effect ( higher growth rates for the secondary subharmonic) as the primary 2D amplitude A increases. At $R=1050$, Fig.(6) illustrates the destabilizing effect of increasing $A$ for $M_{\infty}=0.8$ compared with that for $M_{\infty}=0$. Fig. (6) reveals that the influence of compressibility on secondary subharmonics is not only function of the spanwise wavenumber, but also is function of the primary 2D amplitude. While at $A=.01$ compressibility is stabilizing for large values of $B$ and destabilizing for small values of $B$, it has a destabilizing influence on the secondary subharmonics at $A=.002$ for all range of spanwise wavenumbers. The figure also shows that at very small amplitudes, considerable growth rates exist in a small band of wavenumbers, that extends to larger values as the amplitude increases.

Fig. (7) shows the effect of Reynolds number $R$ on the growth of the secondary subharmonics at $M_{\infty}=0.8$. As $R$ increases, an increase in the growth rates exist at fixed $F$ and $A$. 
To evaluate the overall effect of compressibility on the secondary subharmonics, we can combine the influence of increasing the amplitude $A$ of the primary 2D wave and increasing $R$ for various Mach numbers at fixed F. For comparison purposes, the amplification factor of the subharmonic is calculated using equation (15) from $R_{\text {os }}$ ( onset of the secondary subharmonic instability) to any $R$ downstream. For different Mach numbers, Fig. (8) shows the variation of the growth rates of the secondary subharmonics with $R$ at a spanwise wavenumber $B=.15$. The initial primary 2D amplitude used for these calculations is $A=.001$. Fig. (9) shows the amplification factors decreasing from about 29 at $M_{\infty}=0$. t0 12 at $M_{\infty}=0.8$.

Figs.(8) and (9) indicate that while the growth rates and amplification factors of the secondary subharmonics are decreased by increasing Mach number, the onset of the secondary instability is almost not affected. This is probably due to the combined effect of the upstream shift of the onset of the primary $2 \mathrm{D}$ instability, as well as the growing of the primary amplitude.

\section{CONCLUSION}

We formulated the secondary three-dimensional instability problem for compressible boundary layers. The effect of finite amplitude two-dimensional T-S wave on the growth of three-dimensional subharmonics is investigated for a range of Mach numbers from 0 to 1.2. Numerical results for $F=60 E-6$ show that the local (at fixed $R$ ) effect of compressibility on the secondary subharmonics may be stabilizing or destabilizing depending upon their spanwise wavenumbers, 
as well as the finite amplitude of the primary $2 \mathrm{D}$ wave.However, the overall effect of increasing Mach number is a reduction in the growth rates and amplification factors of the secondary subharmonics, almost with no change in the streamise location where this instability sets in.

\section{ACKNOWLEDGMENT}

This work was supported by NASA Langley Research Center under grant NAG1-729. The author is indebted to P.J. Bobbitt and W.D. Harvey for their interest and support during the course of this work.

REFERENCES

1. Mack, L.M. "Review of Linear Compressible Stability Theory". In Stability of Time Dependent and Spatially Varying Flows. Ed. D.L. Dwoyer and M.Y. Hussaini, Springer-Verlag, N.Y., pp. 164-187, 1987.

2. Erlebacher,G. and Hussaini,M.Y. "Incipient Transition Phenomena in Compressible Flows over a Flat Plate." NASA CR-178111, ICASE Report $86-39,1986$.

3. Erlebacher, G. and Hussaini, M.Y. "Stability and Transition in Supersonic Boundary Layer". AIAA Paper No. 87-1416, 1987. 
4. Herbert, Th." Subharmonic Three-Dimensional Disturbances in Unstable Plane Shear Flows." AIAA Paper No. 83-1759, 1983.

5. Herbert Th., Bertolotti, F.P., and Santos, G.R.," Floquet Analysis of Secondary Instability in Shear Flows." In Stability of Time Dependent \& Spatially varying Flows, Ed. D.L. Dwoyer \& M.Y. Hussaini, Springer-Verlag, N.Y., pp. 43-57, 1987.

6. Nayfeh, A.H." Nonlinear Stability of Boundary Layers". AIAA Paper $87-0044,1987$.

7. E1-Hady N.M. and Nayfeh A.H., " Nonparallel Stability of Compressible Boundary-Layer Flows". AIAA Paper No. 80-0277, 1980 (see a1so VPI\&SU Report No. VPI-E-79.13).

8. Scott, M.R. and Watts, H.A., " Computational Solution of Linear Two-Point Boundary Value Problems Via Orthonormalization". SIAM J. Numer. Anal., Vol. 14, pp. 40-70, 1977.

9. E1-Hady, N.M." On the Effect of Boundary Layer Growth on the Stability of Compressible F1ows." NASA CR-3474, 1981.

10. E1-Hady, N.M.," Secondary Subharmonic Instability of Boundary Layers with Pressure Gradient and Suction". NASA CR-4112, 1988.

11. Orzag, S.A. and Patera, A.T.,"Secondary Instability of Wall Bounded 
Shear Flows. J. Fluid Mech., Vol. 128, 347, 1983.

APPENDIX A

$$
\begin{aligned}
& a_{1}=f\left(g \bar{z}_{1}+\beta \bar{z}_{4}\right)+D\left(f \bar{z}_{3}\right)+g u \bar{F}+D(v \bar{F}) \\
& b_{1}=0 \\
& a_{2}=\left[\bar{G} f+T^{-1} g u-m_{2} \hat{R} \bar{g}(\bar{g}+1 \alpha) t+\hat{R} \beta^{2} t\right] \bar{z}_{1} \\
& +\left[T^{-1} v-\vec{R}^{-1} D(\hat{\mu} t)\right] \bar{z}_{2}+\left[f D U+T^{-1} D u-R^{-1} g D(\hat{\mu} t)\right] \bar{z}_{3} \\
& -\hat{R} \quad t \overline{D Z}_{2}-\hat{R}(m g+\bar{g}) t \overline{D Z}_{3}+\hat{R} \beta\left(m_{1} \bar{g}+i m \alpha\right) t \bar{Z}_{4} \\
& -\hat{R}\left[m_{2} \alpha(i \bar{g}+\alpha) u+\left(i m_{1} \alpha+m \bar{g}\right) D v+D^{2} u\right] \bar{z}_{7} \\
& -\hat{R}(D u+i \alpha v) D\left(\hat{\mu} \bar{z}_{7}\right)+[i(\alpha U-\omega)+v D U] F
\end{aligned}
$$

$$
\begin{aligned}
b_{2}= & {[(g+i \alpha) \bar{f} u+\bar{g} f \bar{u}] z_{1}+(\bar{f} v+f \bar{v}) z_{2}+\left(\bar{f} D u+f(\bar{u}) z_{3}\right.} \\
& +(\bar{v} D u+v D \bar{u}) F
\end{aligned}
$$

$$
\begin{aligned}
a_{z}= & {\left[f \bar{G}+T^{-1}(\bar{g} u+D v)-\hat{R}\left(\bar{g}^{2}+i \bar{g} \alpha-\beta^{2}\right) t\right] \bar{z}_{3} } \\
& +\left[T^{-1} v-m_{2} R^{-1} D(\hat{\mu} t)\right] \bar{z}_{3}-m_{2} \hat{R}_{t} D^{2} \bar{z}_{3}+i(\alpha U-\omega) v \bar{F} \\
& \left.+\left[i T^{-1} \alpha v-m \vec{R}^{-1} D(\hat{\mu} t) \bar{g}\right] \bar{z}_{1}-\hat{R}_{\left(m_{1}\right.} \bar{g}+i \alpha\right) t \bar{z}_{2}-m \vec{R}^{-1} \beta D\left(\hat{\mu}^{t}\right) \bar{z}_{4}
\end{aligned}
$$




$$
\begin{aligned}
& -m_{1} \hat{R} \beta t \bar{z}_{5}-\left\{\hat{R}\left[(i m \alpha+g) D u+(i \bar{g}-\alpha) v+m_{2} D^{2} v\right]\right. \\
& \left.-\hat{R}^{-1} D \hat{\mu}\left(\operatorname{im} \alpha u+m_{2} D v\right)\right\} \bar{z}_{7}-\hat{R}\left(\operatorname{im} \alpha u+m_{2} D v\right) \bar{z}_{8} \\
& b_{3}=1 \alpha(\bar{f} v-f \bar{v}) z_{1}+[(\bar{f} u+f \bar{u}) g+\bar{f} D v+f D \bar{v}] z_{3} \\
& +(\bar{f} v+f \bar{v}) D Z_{3}+[2 v D \bar{v}+1 \alpha(v \bar{u}-\bar{v} u)] F \\
& a_{4}=\left[f \bar{G}+T^{-1} \bar{g} u-\hat{R}\left(\bar{g}^{2}+1 \alpha g-m \beta\right) t\right] \bar{z}_{4} \\
& +\left[T^{-1} v-R^{-1} D(\hat{\mu} t)\right] \bar{z}_{S}-\hat{R}\left[t \bar{z}_{5}-\beta t(g+m \bar{g}) \bar{z}_{l}\right. \\
& \left.-m_{1} \beta t D \bar{z}_{3}-m \beta(1 \alpha u+D v) \bar{z}_{7}\right]+R^{-1} \beta D(\hat{\mu} t) \bar{z}_{3} \\
& b_{4}=(\bar{f} u+f \bar{u}) g z_{4}+(\bar{f} v+f \bar{v}) z_{5} \\
& a_{5}=\left\{f \bar{G}+T^{-1} \bar{g} u-2 \hat{M} \hat{R} D U(D u+1 \alpha v)-\operatorname{Pr}^{-1}\left[\hat{R} t\left(\bar{g}^{2}+2 i \alpha \bar{g}-\alpha^{2}-\beta^{2}\right)\right.\right. \\
& \left.\left.+R^{-1} D(\hat{\mu} D t)\right]\right\} \bar{z}_{7}+[i(\alpha U-\omega) t+v D T] \bar{F}-\hat{M} \bar{g} u \bar{z}_{6} \\
& -\hat{M} v D \bar{Z}_{6}+\left[T^{-1} v-\operatorname{Pr}^{-1}(2 \hat{R} D t+t R \hat{D \mu})\right] \vec{Z}_{8}-\hat{R} \operatorname{Pr}^{-1} t D \bar{Z}_{8} \\
& +\left[1 \propto\left(T^{-1} t-\hat{M} p\right)-2 \hat{M} \mu R^{-1} \bar{g}\left(1 \alpha m_{z} u-m D v\right)\right] \bar{Z}_{1} \\
& -2 \hat{M} R^{-1}[\mu(i \alpha v+D u)+\hat{\mu} t D U]\left(\bar{z}_{2}+\bar{g} \bar{z}_{3}\right) \\
& +\left(T^{-1} D t+f D T-\hat{M} D p\right) \bar{z}_{3} \\
& -2 \hat{M} R^{-1} \mu\left[\left(i m \alpha u+m_{2} D v\right) \overline{D Z}_{3}+m \beta(1 \alpha u+D v) \bar{z}_{4}\right]
\end{aligned}
$$

16 


$$
\begin{aligned}
b_{5}= & \left\{(\bar{f} u+f \bar{u}) g-2 \hat{M} \hat{R}\left[\alpha^{2} \vec{v} v+i \alpha(v D \bar{u}-\bar{v} D u)+m_{2} \alpha^{2} \bar{u} u\right.\right. \\
& \left.+m \alpha^{2}(u \bar{v}+\vec{u} v)+m_{2} D v D \bar{v}+D \bar{u} D u\right] z_{7} \\
& +[i \alpha(\bar{u} t-u \bar{t})+\bar{v} D t+v D \bar{t}] F+(\bar{f} v+f \bar{v}) z_{8} \\
& +\left[i \alpha(t \bar{f}-\bar{t} f)-2 \hat{M} R^{-1} i m_{2} \alpha g(\bar{t} u-t \bar{u})\right] z_{l} \\
& -2 \hat{M} \hat{R}\left(\bar{t} D u+t D \bar{u}+i \alpha(\bar{t} v-t \bar{v})\left(z_{2}+g z_{3}\right)\right. \\
& +(\bar{f} D t+f D \bar{t}) Z_{3}-2 \hat{M} \hat{R}_{2}(\bar{t} D v+t D \bar{v}) D Z_{3}
\end{aligned}
$$

where

$$
\begin{aligned}
& f=T^{-1}\left(\gamma M_{\infty}^{2} p-T^{-1} t\right) \\
& F=T^{-1}\left(\gamma M_{\infty}^{2} z_{6}-T^{-1} z_{7}\right) \\
& \hat{R}=\hat{\mu} R^{-1} \\
& m_{1}=m+1, \quad m_{2}=m+2
\end{aligned}
$$

and the overbar denotes complex conjugate. The overbar in $\bar{\mu}$ and the subscript $r$ in $\alpha_{r}$ are omitted for simplicity $\left(\mu\right.$ is $\bar{\mu}$ and $\alpha$ is $\left.\alpha_{r}\right)$. 


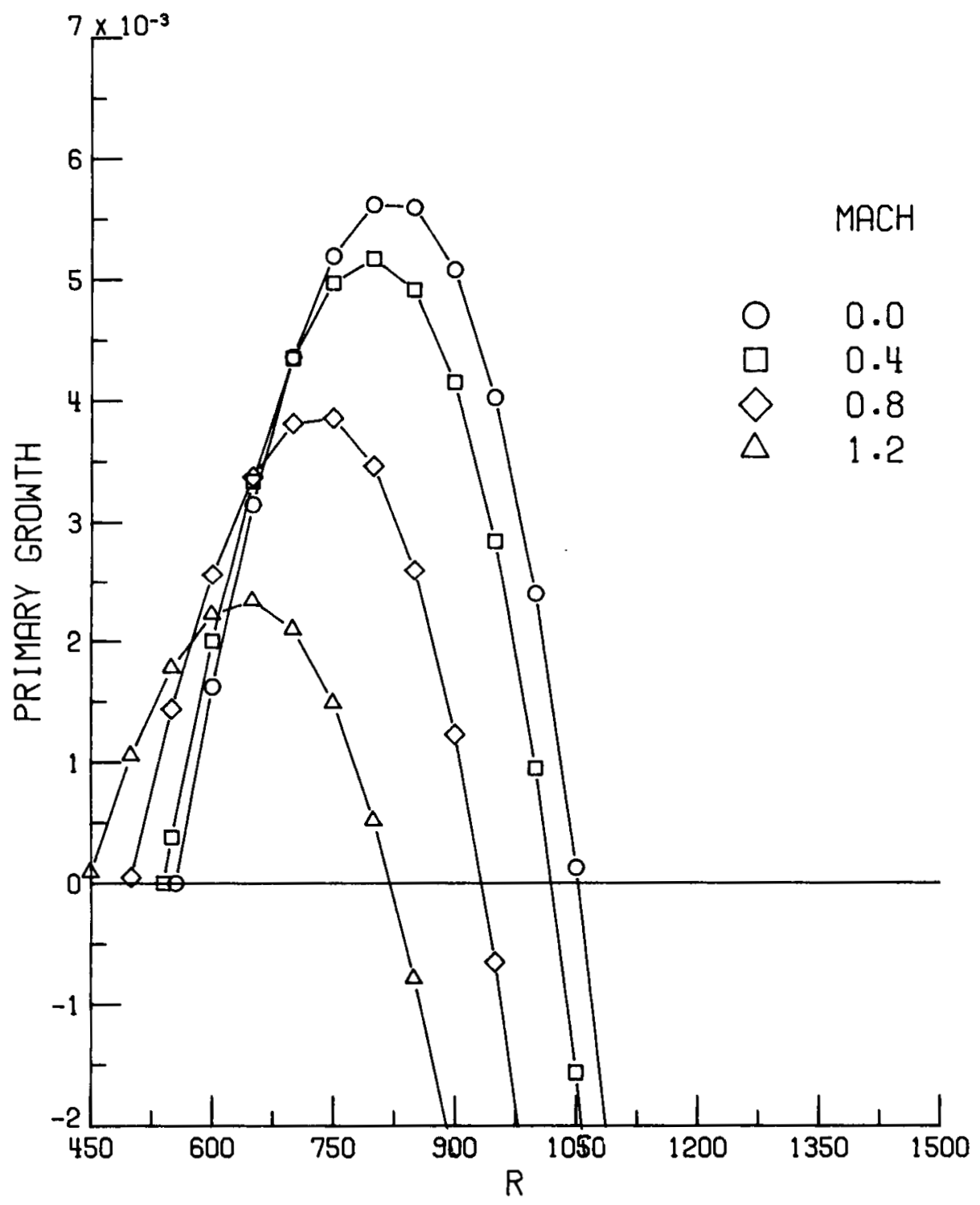

Fig. 1 Growth rates of the primary $2 D$ wave $(F=60 E-6)$ at different Mach numbers. 


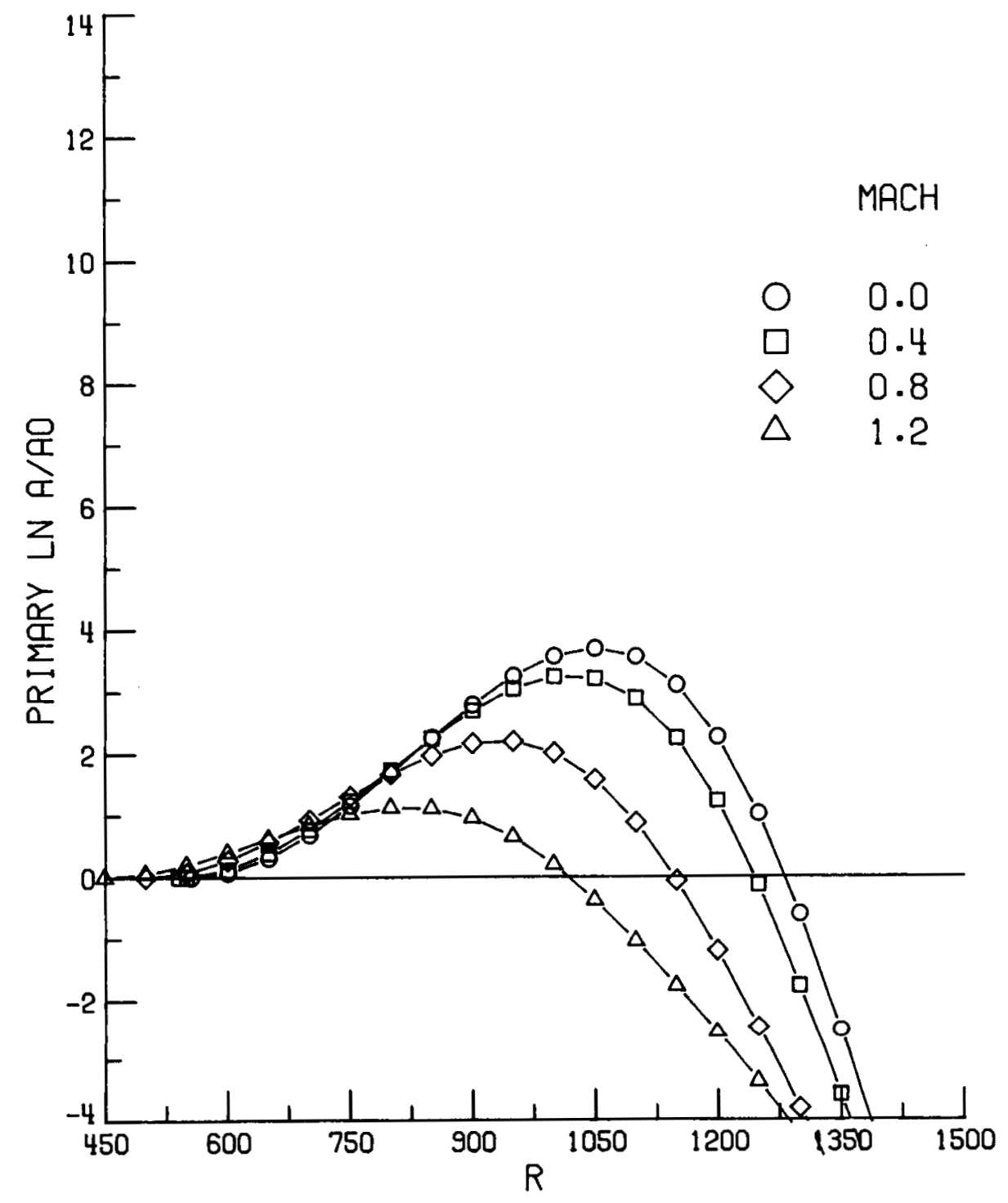

Fig. 2 Amplification factors of the primary 2D wave $(F=60 E-6)$ at different Mach numbers. 


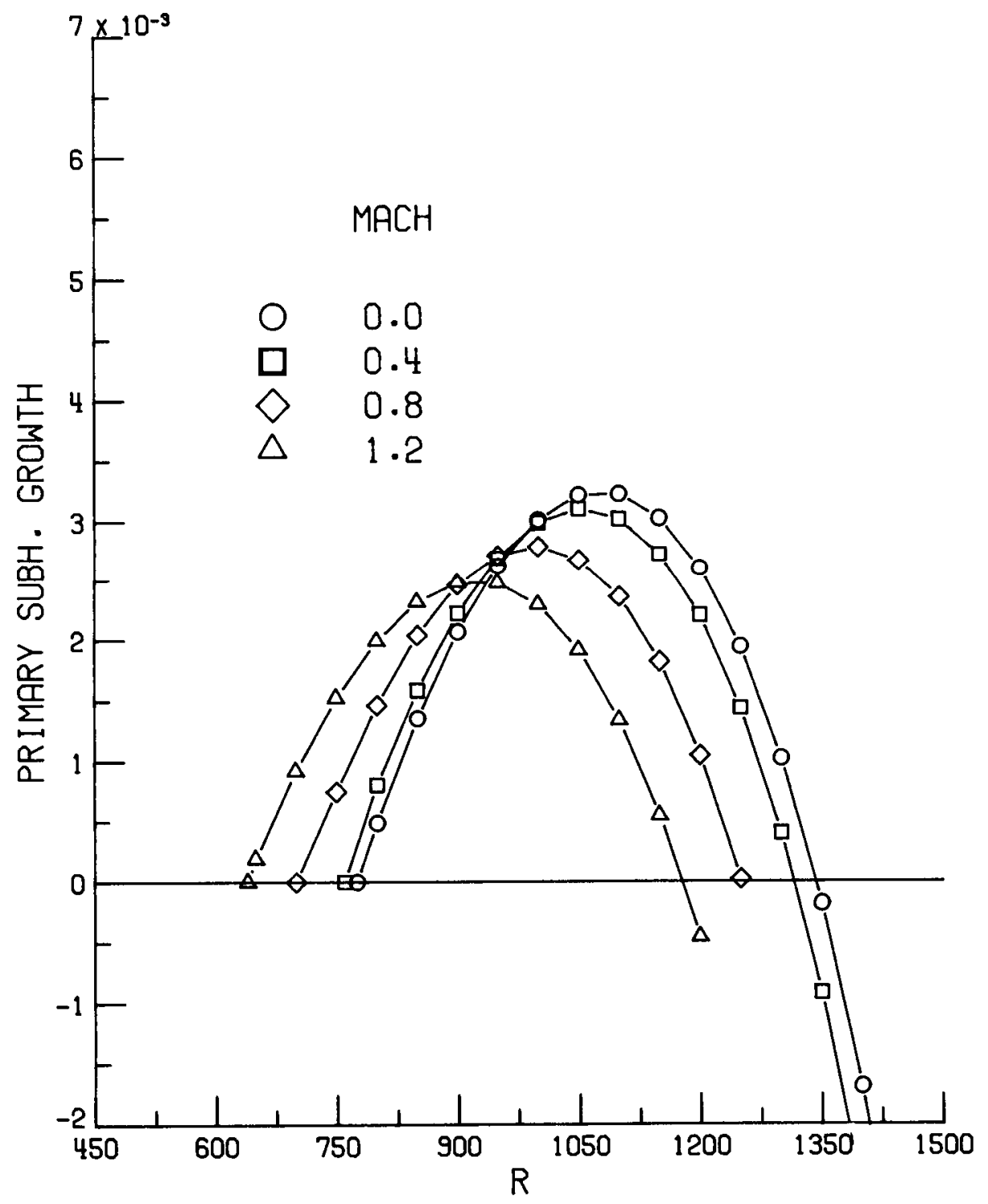

Fig. 3 Growth rates of the primary 3D subharmonic wave $(\mathrm{F}=30 \mathrm{E}-6$ and $\mathrm{B}=.12$ ) at different Mach numbers. 


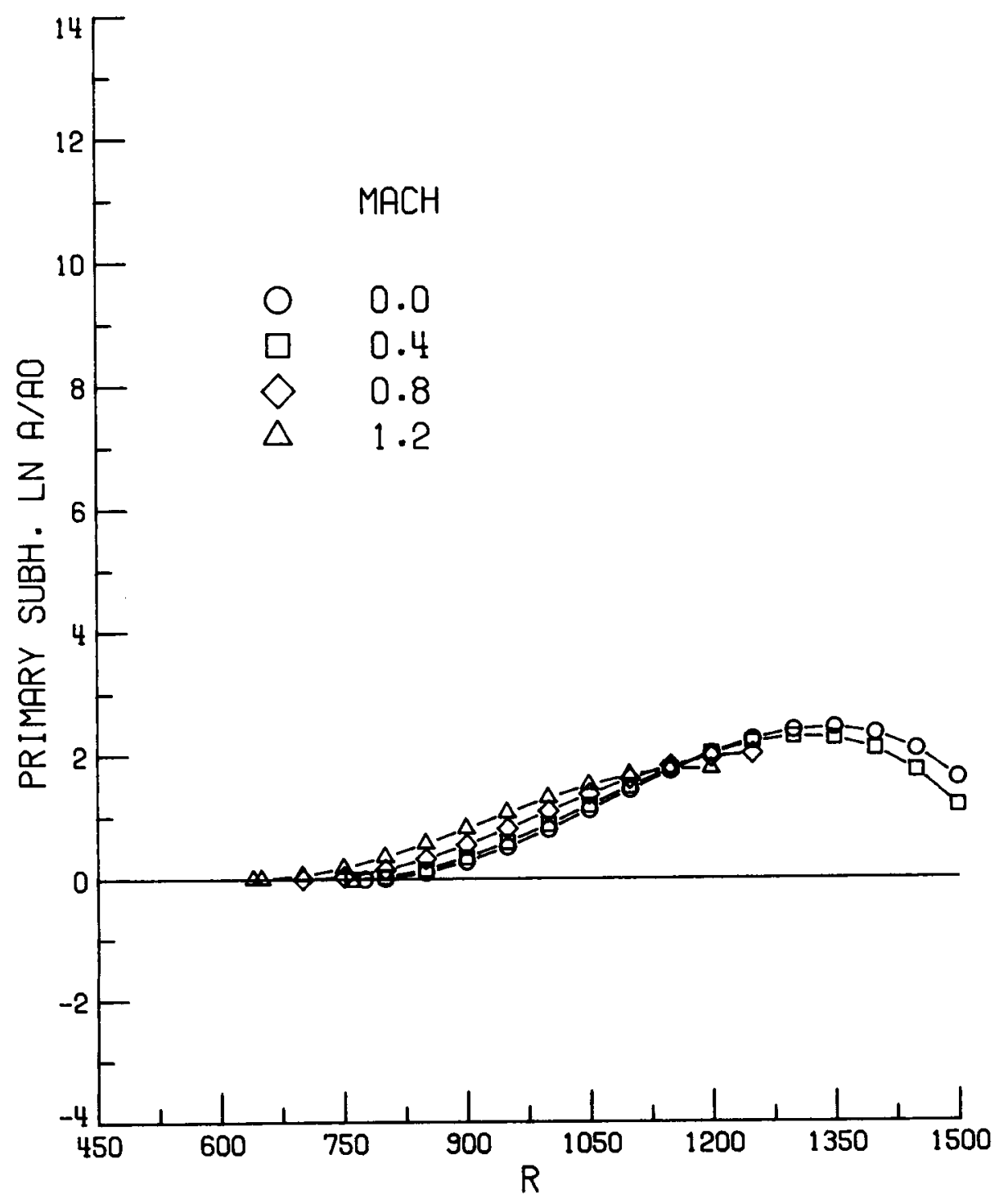

Fig.4 Amplification factors of the primary 3D subharmonic wave $(F=30 E-6$ and $B=.12)$ at different Mach numbers. 


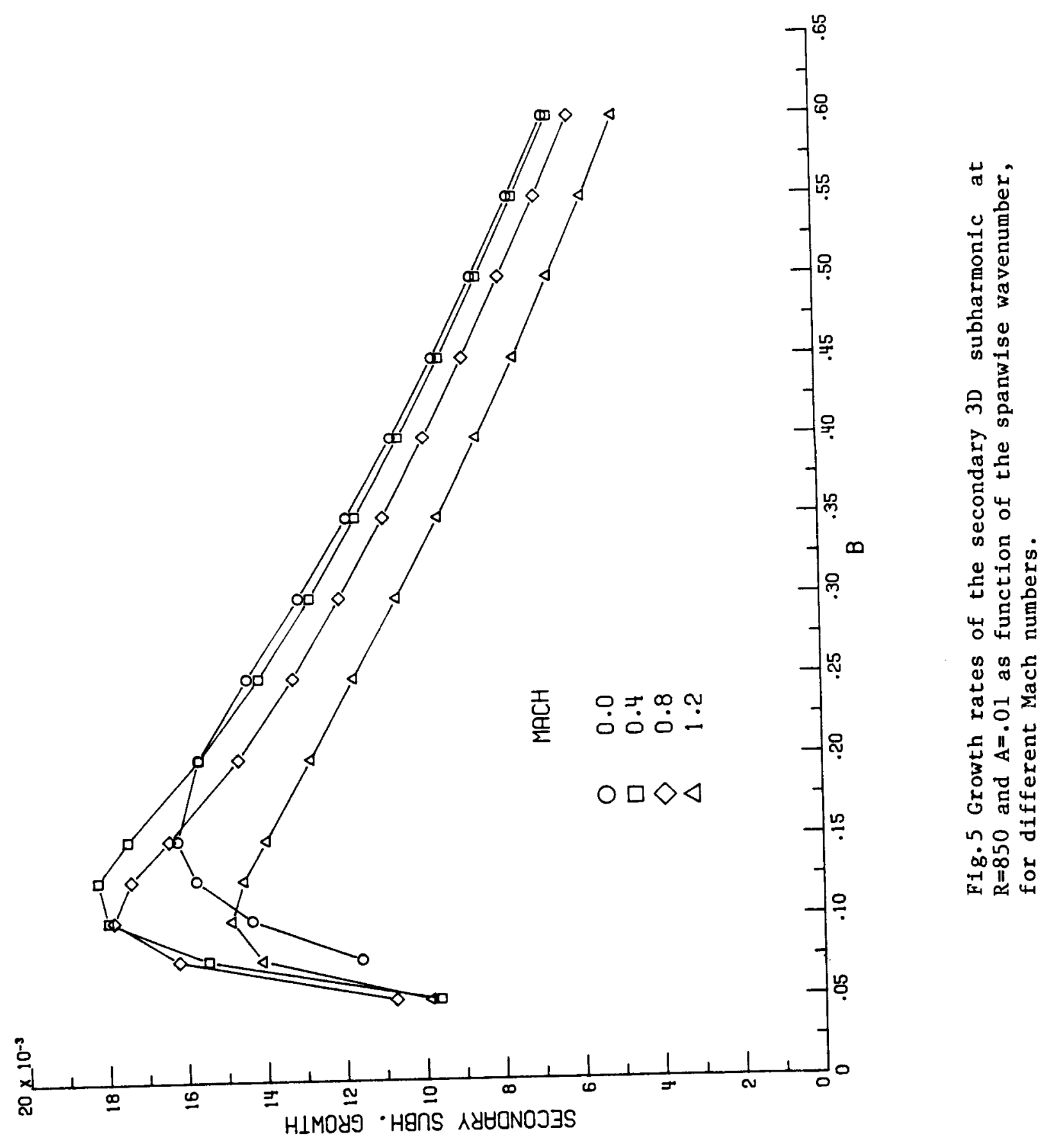




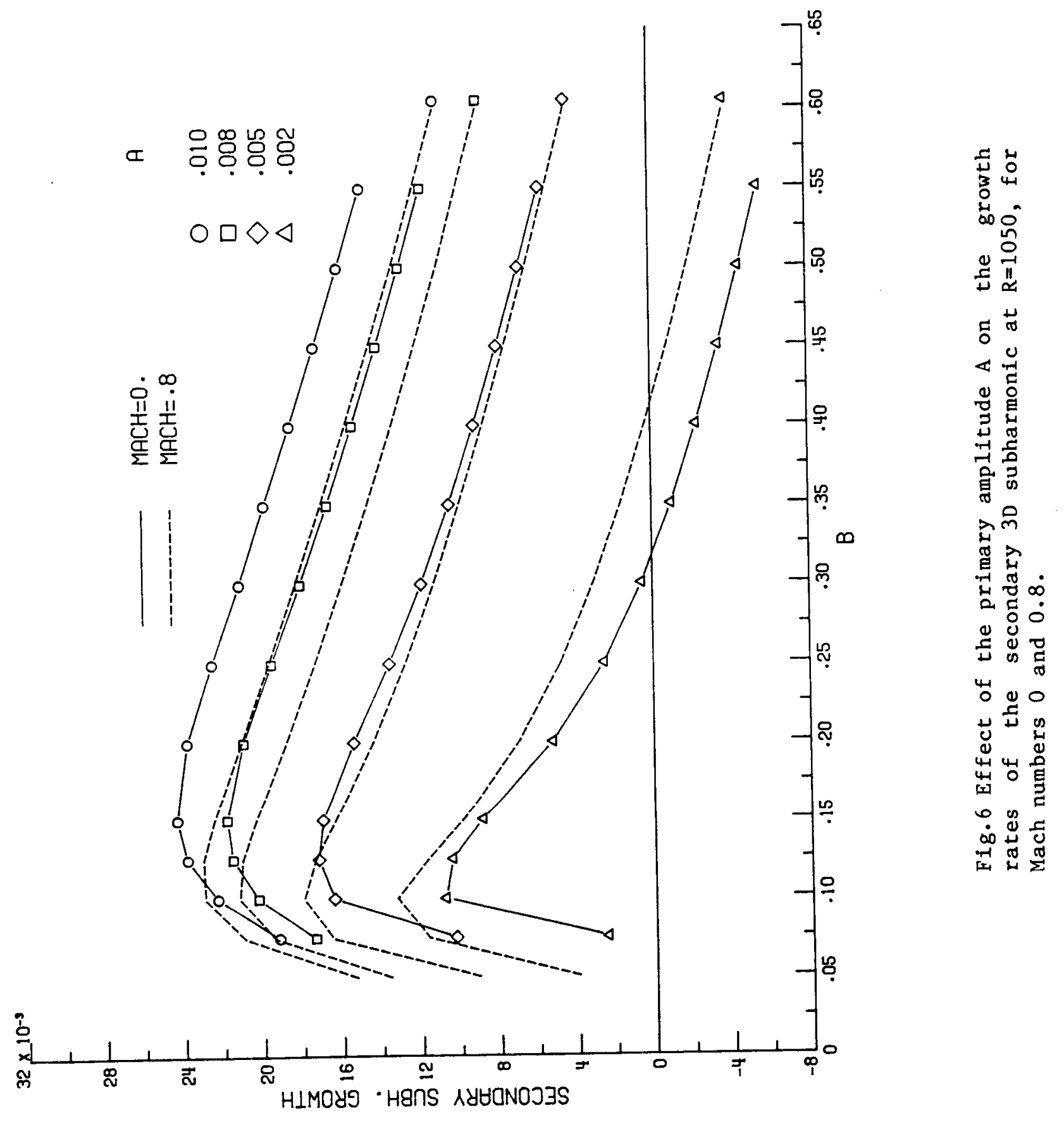




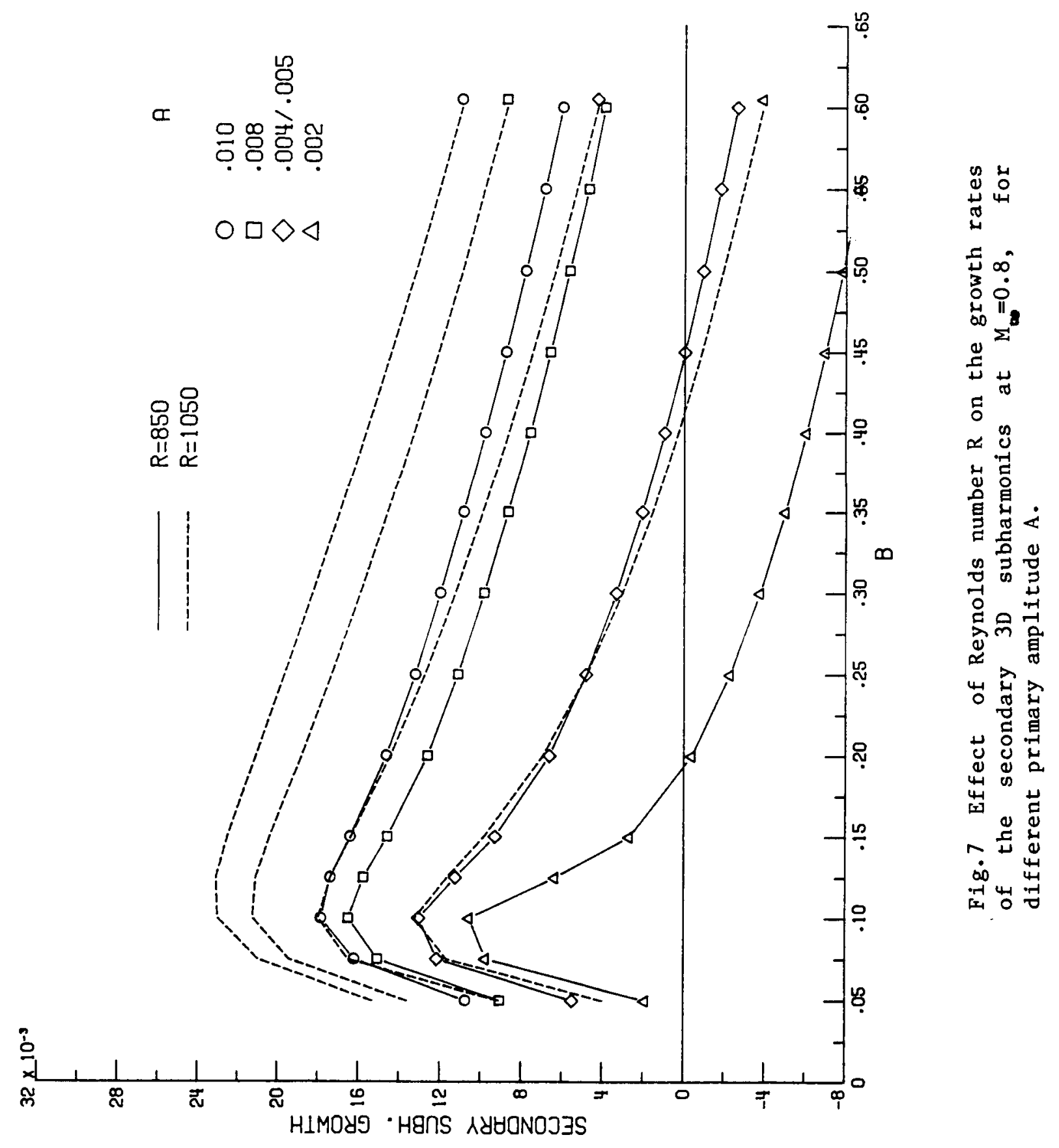




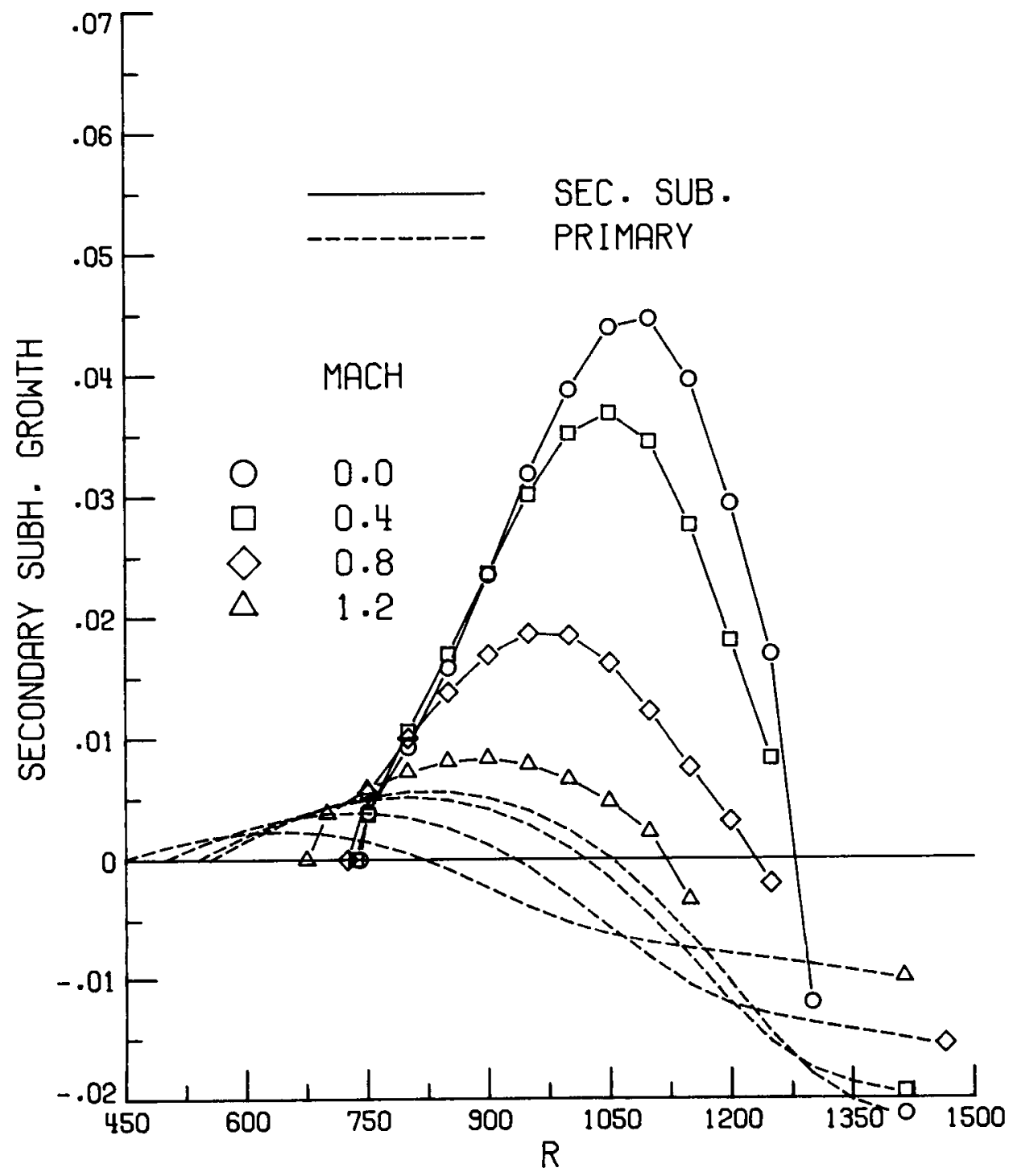

Fig. 8 Variation of the growth rates with $R$ of $a$ secondary $3 D$ subharmonic at $B=.15$ for different Mach numbers. 


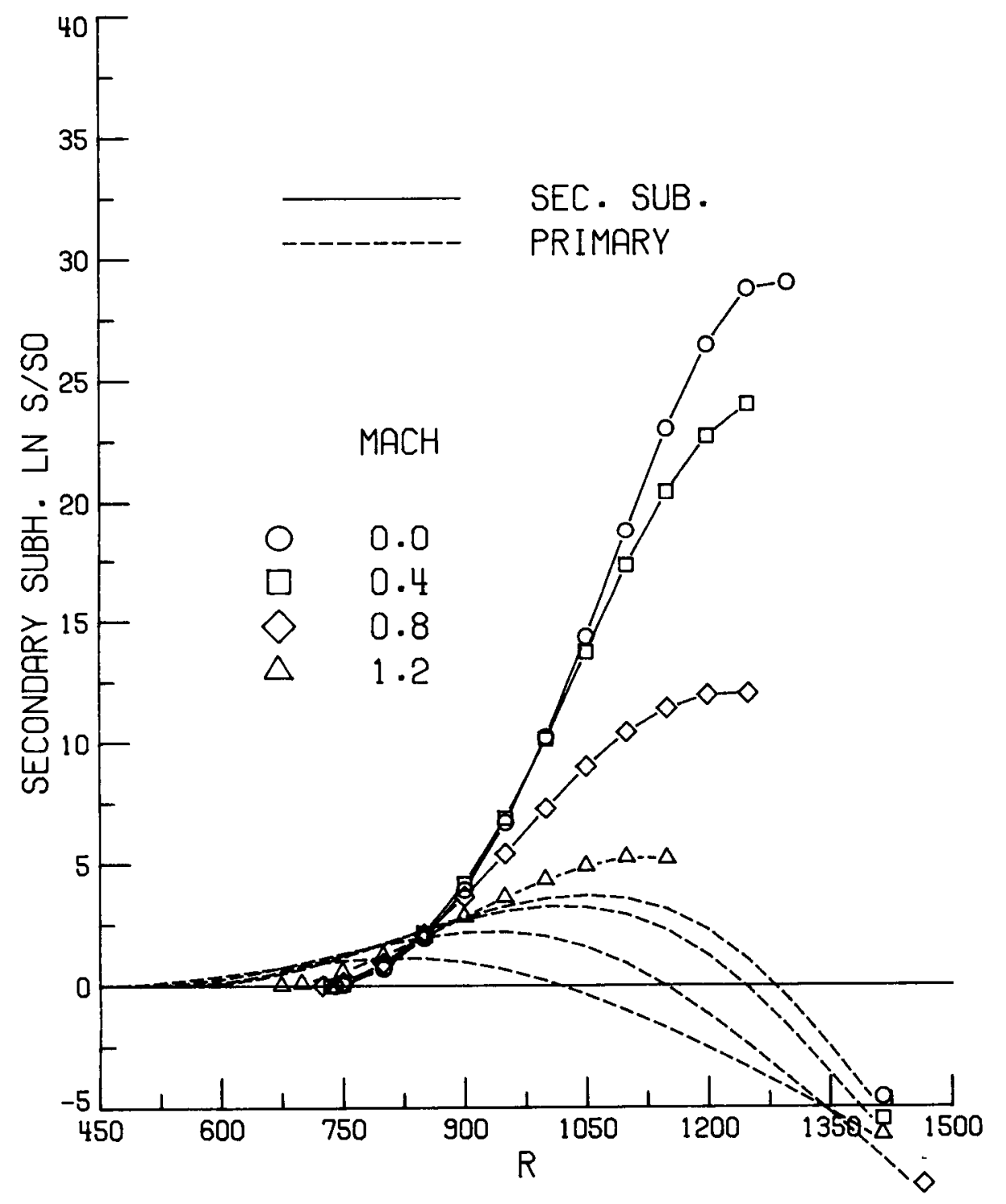

Fig. 9 Variation of the amplification factors with $R$ of a secondary 3D subharmonic at $B=.15$ for different Mach numbers. 


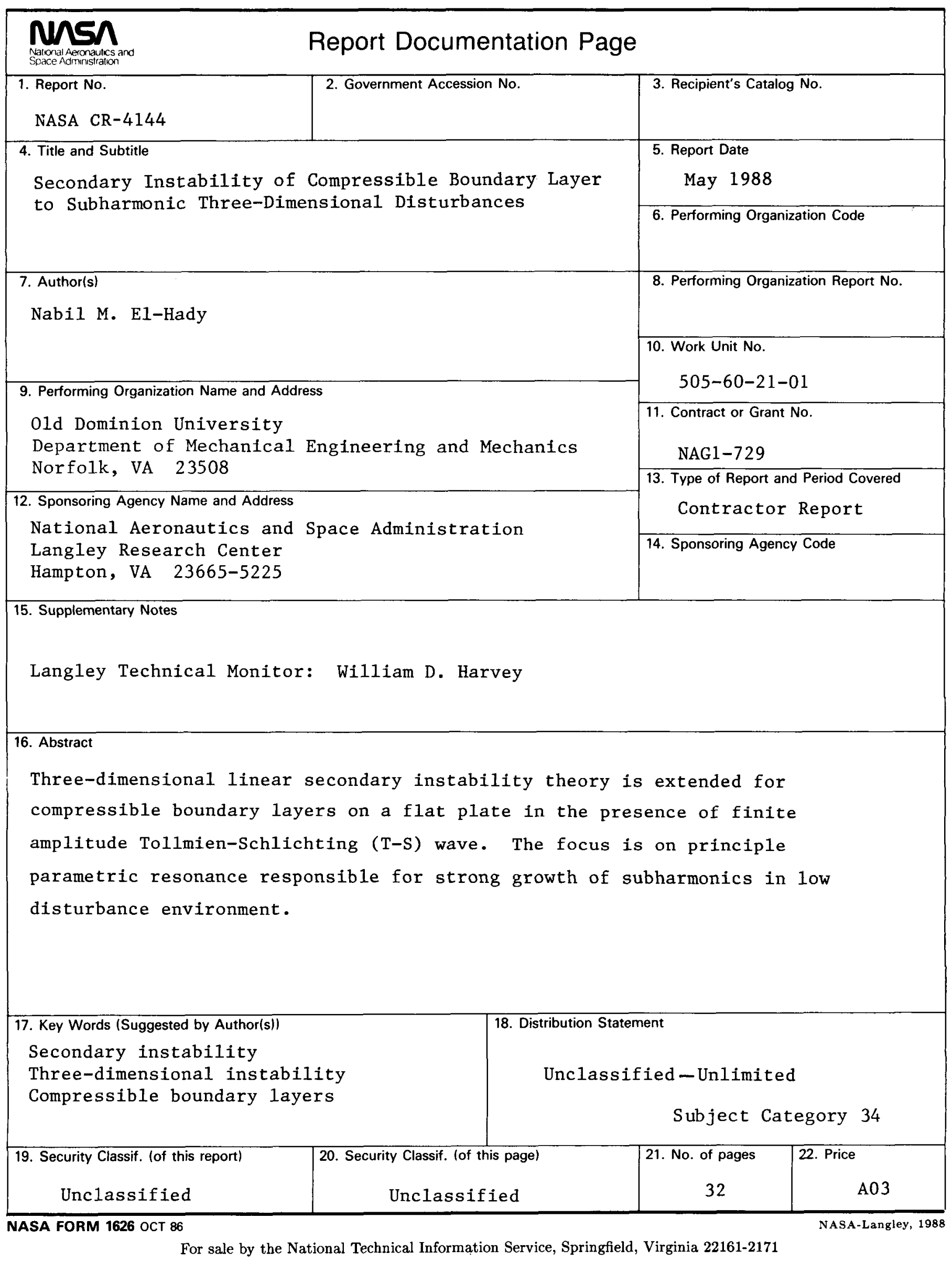

